\title{
Comércio internacional, blocos econômicos e meio-ambiente: uma avaliação de impactos utilizando equilíbrio geral aplicado
}

\author{
Willian Mattes Gonçalves* \\ Flavio Tosi Feijó**
}

\begin{abstract}
Resumo: Este trabalho analisa o efeito dos acordos internacionais de livre comércio sobre o meio-ambiente. A partir de 1950, as relações comerciais ganharam um novo impulso e passaram a desempenhar um papel fundamental nas economias nacionais. Ao mesmo tempo, o crescente número de acordos de integração regional pretendeu tornar os países menos restritivos ao comércio. O procedimento metodológico para a avaliação dos impactos ambientais dos acordos de liberalização comercial internacional consistiu na ponderação das variações do produto dos setores da indústria pelos respectivos índices lineares de toxicidade humana aguda (ILTHA) antes e após a simulação de redução total de tarifas de importação e exportação para três cenários de acordos de livre-comércio: Brasil-Resto do Mercosul; Brasil-NAFTA e Brasil-UE. Assume-se neste trabalho que a liberalização comercial elimina completamente as tarifas comercias. Os resultados mostram que existem ganhos ambientais potenciais decorrentes do comércio internacional. No entanto, esses ganhos dependem do grau de alteração causado pelo comércio na participação dos setores no produto e do grau de intensidade de poluição de cada um deles.
\end{abstract}

Palavras-chave: livre-comércio; integração regional; meio-ambiente.

Classificação JEL : F18, Q56.

\footnotetext{
* Doutorando em Economia pelo PPGE-UFPB e Professor assistente do Departamento de Economia/UFPB

** Doutor em Economia pela UFRGS e Professor Associado da Faculdade de Economia UFRGS e do PPGE/UFRGS
} 


\section{Introdução}

A economia mundial sofreu profundas modificações ao longo dos últimos cinqüenta anos. Tais mudanças foram notadamente influenciadas pela dinâmica de crescimento da população humana, pelo aprimoramento das tecnologias da informação, pela nova postura adotada nas relações internacionais, incluindo relações comerciais, e mais recentemente pela difusão crescente dos debates e estudos sobre as inter-relações dos processos produtivos e seus impactos ambientais regionais e globais. Em termos acadêmicos, houve notadamente um aumento de interesse em torno da problemática existente entre os impactos do comércio internacional sobre o meio-ambiente, refletido pelo recrudescimento de discussões científicas que integram parte de uma proposta de pesquisa mais ampla, a qual visa estudar a interferência da ação humana sobre as alterações climáticas globais e suas consequências.

As atividades produtivas impactam o meio-ambiente por meio da utilização contínua de recursos naturais, renováveis ou não, e das emissões residuais e descartes resultantes de seus processos. Particularmente, houve uma notável intensificação da escala de produção com o advento da Revolução Industrial, pois as novas técnicas permitiram a produção em larga escala e a expansão do consumo. Desde então, inúmeros problemas se conjugaram à expansão da população mundial e suas novas demandas, cujos efeitos podem estar em vias de afetar os limites da estabilidade ecossistêmica do planeta.

A degradação ambiental causada pelas atividades humanas tem se refletido principalmente em poluição atmosférica; poluição terrestre; poluição de cursos de água, mares e oceanos; desflorestamentos; sobre-pesca; destruição da camada de ozônio et caetera. Da mesma forma, as conseqüências daí resultantes são variadas, mas possuem em comum a capacidade de afetar sobremaneira o bem-estar agregado dos indivíduos, tanto pelos seus impactos diretos sobre a saúde humana como pelos custos resultantes do número crescente de catástrofes e desastres naturais, possivelmente decorrentes de causas antropogênicas.

O fenômeno da integração regional internacional é relativamente recente na história econômica e pode ser atribuído à formalização de Acordos Preferenciais de Comércio (APC's) ainda no século XX. Desde 1934, a partir da negociação de APC entre o Reino Unido e suas antigas colônias, os processos de integração regional se intensificaram de modo substancial, resultando em crescente formação de blocos econômicos regionais com diferentes níveis de integração econômica.

Dada a relevância desses temas, a velocidade das mudanças econômicas e o consenso de que o comércio internacional é benéfico ao bem-estar econômico, torna-se necessário que se investigue também a magnitude dos impactos do comércio internacional sobre o meio-ambiente. Por isso, pretende-se neste trabalho avaliar as potenciais conseqüências da liberalização comercial internacional a partir das variações setoriais da produção industrial e de suas potencialidades toxicológicas. Com esse objetivo, utilizou-se um modelo aplicado de equilíbrio geral, o GTAP (General Trade Applyed Project) para realizarem-se 
as simulações na base de dados e o Índice Linear de Toxicidade Humana Aguda (ILTHA), do Sistema de Projeções da Poluição Industrial (IPPS), do Banco Mundial.

O procedimento metodológico para a avaliação dos impactos ambientais dos acordos de liberalização comercial internacional consistiu na ponderação das variações do produto dos setores da indústria pelos respectivos ILTHA's antes e após a simulação de redução total de tarifas de importação e exportação entre o Brasil e os parceiros analisados. Assume-se, neste caso, que a liberalização comercial elimina completamente as tarifas comercias.

Na próxima seção, serão apresentadas algumas considerações a respeito do comércio internacional e do fenômeno da integração econômica regional e seus impactos sobre o meio ambiente. Na terceira seção, apresentam-se o instrumental analítico a ser utilizado neste trabalho - a saber, a teoria do equilíbrio geral - um modelo aplicado de equilíbrio geral computável - o GTAP - e o Sistema de Projeções da Poluição Industrial (IPPS). Por fim, a última seção apresenta e discute os resultados encontrados.

\section{Comércio internacional, integração regional e meio ambiente}

Modernamente, o estabelecimento de APC's pode se dar através da criação de área de livre comércio ou união aduaneira (Krugman e Obstfeld, 2003 , p. 243). No primeiro caso, as trocas referentes ao comércio internacional intrabloco são livres de tarifas, fato que implica livre trânsito comercial entre os parceiros da área. No segundo caso, a formação de uma união aduaneira implica uma coordenação comum da política tarifária externa, havendo necessidade de consenso sobre a aplicação de uma tarifa externa comum sobre os bens importados.

De acordo com WTO (2000), ao longo dos últimos dez anos ocorreram importantes modificações quantitativas e qualitativas nos arranjos institucionais dos acordos de integração regional, mas principalmente o reconhecimento de que a integração regional requer mais do que a redução de tarifas e quotas. Além disso, nota-se também uma reorientação de um "regionalismo-fechado" para uma postura pró-ativa com o comércio internacional extrablocos. Outra característica que se observa é a formação de blocos compostos por países em diferentes níveis de desenvolvimento econômico, cujos exemplos mais notáveis são o NAFTA (North Atlantic Free Trade Agreement), instituído em 1994 por EUA, Canadá e México, e a extensão dos acordos europeus a países da Europa Oriental, como o acordo de união aduaneira entre União Européia e Turquia.

Os principais aspectos estratégicos, econômicos e políticos relacionados com a implementação de blocos regionais podem ser resumidos pelo excerto:

No country is immune from the effects of regionalism as it shapes world economic and political relationships and influences 
the development of the multilateral trading system, and all countries face policy choices concerned with regionalism. Should they enter a regional integration agreement? With what other coutries? What measures should be implemented - simple trade liberalization or deeper harmonization of domestic policies?

(WTO, 2000, p. 5)

Essas questões têm sido amplamente investigadas no que diz respeito aos seus benefícios e prejuízos efetivos, uma vez que os países diferem substancialmente em seus objetivos econômicos e políticos. Krugman (1991) demonstrou que a regionalização reduz o bem-estar econômico mundial. Oladi e Beladi (2008), utilizando um modelo em que o processo de decisão política de formação de blocos econômicos é endogenamente determinado, argumentam que um subconjunto de países, quando considera a formação de um bloco, leva em conta a possibilidade de formação futura de outros blocos, sem assumir a efetividade do comprometimento de qualquer país com qualquer bloco.

O resultado notável trazido por Oladi e Beladi (2008) mostra que nenhum bloco econômico é estável, e advertem que os blocos econômicos regionais não devem ser vistos como solução dos problemas do comércio internacional, tanto por não serem uma alternativa maximizadora de bem-estar, como pela sua natureza inerentemente instável. A formação de blocos regionais deve ser encarada como um passo em direção à criação de um bloco global de comércio através da sustentação de contínuas rodadas de negociação para se reduzirem os entraves ao comércio internacional, tais quais as promovidas pela OMC. Em suma, as nações maximizadoras de bem-estar devem expandir sua área de livre comércio para se integrarem ao conjunto total de países, onde predominaria o equilíbrio. Outro argumento que contribui para o esforço em direção ao livre comércio global é o estímulo à convergência de renda. Nesse sentido, BenDavid e Bohara (1997) mostram que países que procederam a uma extensiva liberalização comercial entre si experimentaram significante convergência de renda. Os autores atribuem a convergência possivelmente aos canais descritos por Hecksher (1919) e Ohlin (1933) da teoria clássica do comércio internacional, à proposição de equalização dos preços de fatores (Samuelson, 1948, 1949; Helpman e Krugman, 1985), ou ao papel de disseminação tecnológica do comércio internacional que afeta o processo de crescimento econômico (Baumol et al., 1989).

Em suma, as discussões sobre liberalização comercial se concentram preponderantemente na avaliação dos custos e benefícios advindos das reformas e dos mecanismos institucionais para assegurá-la. Admite-se, no curto prazo, a inevitabilidade de que a existência de diferenciais de competitividade econômica entre países ocasione importantes custos às economias nacionais por meio da tendência baixista, em termos de produto e emprego, sofrida pelas atividades menos eficientes diante de competidores internacionais mais eficientes. Porém, em longo prazo, os ganhos podem ser basicamente de dois tipos: a redução de preços aos consumidores - e aumento de variedade e qualidade de produtos - e os ganhos de eficiência trazidos pelo processo de ajustamento, em termos de 
tecnologia, aprendizagem, pesquisa e desenvolvimento etc.

$\mathrm{O}$ entendimento prevalecente nas discussões sobre comércio internacional considera que existem efeitos diversos da abertura comercial sobre o meio-ambiente, de forma que o comércio internacional tanto pode se tornar um potencializador de degradações ambientais como pode alterar o nível de competitividade entre países na medida da assimetria de suas políticas ambientais. As duas hipóteses predominantes na literatura, atualmente, postulam que a alocação de indústrias ambientalmente nocivas entre países pode estar sujeita a condicionantes em termos de dotação de fatores ou do grau de exigência das normas legais que regulamentam as políticas ambientais. Estas duas hipóteses são conhecidas como hipótese da dotação de fatores (HDF) e hipótese dos portos de poluição (HPP).

A $H D F$ parte da teoria clássica do comércio internacional para inferir que as vantagens comparativas entre países desempenham um papel importante na determinação da localização de indústrias intensivas em poluição. Assumese que indústrias produtoras de bens ambientalmente sensíveis são intensivas em capital. Decorre dessa pressuposição a tendência de que essas indústrias concentrem-se em países com maiores disponibilidades de capital (países ricos) e reduzam sua participação em países onde esse fator é relativamente escasso.

Por outro lado, a HPP sugere que existem elementos institucionais, precisamente jurídicos, capazes de alterar as vantagens comparativas entre países. Em suma, a assimetria do grau de rigidez e do nível de exigência das normas que regulamentam a política ambiental pode afetar os custos de produção, isto é, o mecanismo de transferência dos custos da política ambiental distorce as vantagens comparativas e, dessa forma, a especialização produtiva entre países. Logo, quanto mais altos os padrões e níveis de exigência da política ambiental, tanto maiores serão os custos de produção necessários para honrá-la.

Nesse sentido, observa-se que países de alta renda (intensivos em capital) possuem legislações ambientais mais rígidas, tornando mais custosa a produção de bens ambientalmente nocivos e reduzindo a vantagem comparativa desses países nesse tipo de produção. Em última instância, esse argumento vai de encontro às conclusões decorrentes da $H D F$, pois demonstra uma superposição de efeitos: de um lado, a dotação relativa de fatores entre países e, por outro, a distorção causada pelos custos advindos do cumprimento de normas de políticas ambientais mais ou menos rígidas.

No entanto, existem divergências quanto à sustentação empírica dessa última hipótese, uma vez que existe uma multiplicidade de fatores que pode também afetar as decisões de investimento, e não tão somente os custos de adequação às regulamentações ambientais. Argumenta Bommer (1999) que a liberalização comercial per se pode aumentar a probabilidade de ocorrência de realocações industriais estratégicas em virtude do rent-seeking. Bommer (1999) ainda ressalta que as assimetrias informacionais entre regulador e regulado têm sido constantemente negligenciadas nas pesquisas empíricas mas podem desempenhar um papel importante na validação dessa hipótese. Os órgãos reguladores, ao selecionar os padrões ambientais, desconhecem em que medida 
suas ações afetarão as firmas e, devido à heterogeneidade dessas últimas, a capacidade delas de se adequarem aos padrões mais elevados de exigências ambientais. Da mesma forma, argumenta que o governo está sujeito a um processo de "otimização política" ao equacionar interesses de grupos de pressão, onde tanto mais flexível será quanto maiores forem as perdas potenciais em termos de produto e emprego. Nesse sentido, Hansen e Prusa (1997) sustentam, por exemplo, que as decisões da US International Trade Comission (ITC) são significativamente influenciadas por fatores políticos e econômicos. Xing e Kolstad (1998) corroboram fracamente a HPP a partir de evidências para a indústria química norte-americana. Os resultados encontrados sugeriam que um país com legislação ambiental mais branda estaria apto a atrair US\$ 0,27 milhão proveniente da indústria química norte-americana para cada ponto percentual permitido de aumento relativo nas emissões de dióxido de enxofre $\left(\mathrm{SO}_{2}\right)$.

Em sentido oposto, Repetto (apud WTO, 1999, p. 40) assevera que, embora os países em desenvolvimento tenham recebido mais de $45 \%$ do investimento direto estrangeiro realizado pelos Estados Unidos, a sua participação em indústrias ambientalmente sensíveis é consideravelmente pequena, contabilizando apenas $5 \%$ desse montante em investimentos naquele tipo de indústria, contra $24 \%$ em países desenvolvidos. Eskeland e Harrison (1997) estudaram os influxos de investimentos diretos estrangeiros em países em desenvolvimento, entre eles México e Venezuela, durante os anos 1980, e argumentam que nenhuma evidência pode ser levantada a respeito de um "viés poluidor" dos investimentos estrangeiros.

Otrabalho de Grossman e Krugman (1998)introduziu metodologicamente a decomposição dos efeitos do comércio internacional sobre o meio-ambiente em três componentes interativos - quais sejam efeito escala, efeito técnica e efeito composição. O efeito composição consiste nas alterações provocadas pelo comércio decorrentes das vantagens comparativas entre países, tais como preconizadas pela teoria clássica do comércio internacional, isto é, a abertura comercial tende levar à especialização da produção na qual um país é mais eficiente. Em última análise, o país passará a importar alguns produtos em detrimento da opção pela produção interna. Esse efeito produzirá uma recomposição da participação dos setores econômicos na produção interna total do país. Logo, espera-se que o efeito composição seja benéfico ao meio-ambiente quando a especialização se der em setores menos poluidores, e vice-versa. Como sugere WTO (1999, p. 3), o comércio internacional está relacionado com uma realocação dos problemas de poluição ao redor do mundo, dada a impossibilidade de especialização de todos os países em indústrias limpas. O efeito escala capta os impactos do aumento da produção, induzido pelo aquecimento da atividade econômica, sobre a poluição. É evidente que o aumento do produto intensifica caeteris paribus a demanda por insumos, incluindo recursos naturais e poluição. No entanto, as evidências empíricas sugerem que o aumento de renda ocasionado pelo comércio eleva pari passu a disposição a pagar dos consumidores por bens menos danosos ao meioambiente, e as suas exigências relativas a métodos de produção ecologicamente 
mais corretos. Diante disso, a internalização dos custos ambientais por parte das firmas opera como um estímulo ao desenvolvimento de novas técnicas e tecnologias adequadas ao novo quadro institucional, traduzido pelo comportamento dos consumidores. A esse movimento de adequação tecnológica dá-se o nome efeito técnica. Esse último efeito resultará em produção mais limpa e relativamente menos intensiva em consumo de recursos e insumos. incluindo recursos naturais e poluiçtesifica a demanda por insumos ela teoria cl originrcos jur

O trabalho de Antweiler, Copeland e Taylor (1998), seguindo a metodologia de Grossman e Krugman (1993), investiga os impactos da abertura comercial sobre o meio ambiente através de estimativas de dados de painel de emissões de dióxido de enxofre $\left(\mathrm{SO}_{2}\right)$ referentes a 44 países, entre 1976 e 1996. Os resultados obtidos sugerem que o comércio internacional tem um impacto relativamente pequeno sobre a poluição quando altera a composição e, conseqüentemente a intensidade de poluição por setor, no produto nacional. No que diz respeito aos efeitos escala e técnica, o comércio internacional implica uma redução líquida da poluição. $\mathrm{O}$ efeito total das estimativas para o caso de dióxido de enxofre mostra que o livre comércio tende a ser ambientalmente desejável, levando a um aumento da concentração de poluição em $0,3 \%$ decorrente de um aumento de $1 \%$ na atividade econômica, e uma redução de $1,4 \%$ na concentração de poluição decorrente do efeito técnica, obtendo-se um resultado líquido total de $1,1 \%$ de queda nas concentrações daquele poluente.

\section{Teoria de equilíbrio geral e instrumentos metodológicos}

A teoria do equilíbrio geral pode ser compreendida como uma extensão do modelo de análise de oferta e demanda de um mercado isolado para o caso de $n$ mercados. Trata-se, portanto, de uma tentativa de relaxarem-se as hipóteses inerentes às análises de equilíbrio parcial, quando se assume que os preços dos demais mercados, que não aquele em análise, sejam constantes. Em equilíbrio geral, todos os preços são variáveis e a condição de equilíbrio requer que esses preços se ajustem às condições de oferta e demanda em seus respectivos mercados para que todos os mercados se equilibrem simultaneamente. Esse fato implica a necessidade de levarem-se em consideração as características de cada mercado isoladamente, e também as interações existentes entre todos os mercados.

Em análises de equilíbrio parcial, a suposição predominante é a de que o setor em análise é livre de influências do resto da economia, enquanto que nas análises de equilíbrio geral, supõe-se que as influências exclusivamente "não-econômicas", e, portanto, além dos limites da análise econômica, sejam mantidas constantes. Logo, o poder preditivo dos modelos de equilíbrio geral depende do grau de independência existente entre as variáveis econômicas e não-econômicas (Mckenzie, 1989, p. 1).

Em uma economia de trocas puras supõe-se que os agentes sejam formados exclusivamente por consumidores com preferências bem-comportadas 
(descritas por uma função-utilidade, $\phi_{i}$ ) e uma dotação inicial de $n$ bens, $\omega_{i}$. Eles alocarão os seus recursos de forma a maximizar $\phi_{i}$ sujeitos a $\omega_{i}$. Sendo a quantidade do bem $j$ possuída pelo agente $i$ denotada por $x_{i}{ }^{j}$, a sua cesta de bens será $\mathrm{x}_{\mathrm{i}}=\left(\mathrm{x}_{i}{ }^{1}, \ldots, \mathrm{x}_{i}{ }^{n}\right)$. Uma alocação física factível somente será possível quando, e se:

$$
\sum_{i=1}^{n} x_{i} \leq \sum_{i=1}^{n} \omega_{i}
$$

Para o caso de dois agentes (A e B) e dois bens (bem 1 e bem 2), por exemplo, temos que a quantidade total do bem 1 é dada por $\omega^{1}=\mathrm{w}_{\mathrm{A}}{ }^{1}+\mathrm{w}_{\mathrm{B}}{ }^{1} \mathrm{e}$, analogamente, a quantidade total do bem 2 por: $\omega^{2}=\mathrm{w}_{\mathrm{A}}{ }^{2}+\mathrm{w}_{\mathrm{B}}{ }^{2}$. A ilustração gráfica das dotações, preferências e alocações pode ser representada em um plano bidimensional com largura $\omega^{1}$ e altura $\omega^{2}$, conhecido como caixa de Edgeworth.

Assumindo-se, para o caso de uma economia de múltiplos agentes, que $i$ agentes são tomadores de preços e a existência de um vetor de preços $p^{*}=\left(p_{1}\right.$, $\ldots, \mathrm{p}_{\mathrm{n}}$ ), pode-se admitir que eles irão se deparar com o seguinte problema de maximização: $\max \phi_{\mathrm{i}}\left(x_{i}\right)$ s.a. $\mathrm{px}_{\mathrm{i}}=\mathrm{p} \omega_{\mathrm{i}}$. Em palavras, espera-se que maximizem sua utilidade sujeitos a restrições de preços (e, portanto, ao valor de mercado) de sua dotação inicial de bens. $\mathrm{O}$ vetor de preços que equilibra todos os mercados leva à condição conhecida como equilíbrio walrasiano, onde inexistem excessos de demanda ou oferta. Varian (1992, p. 316) mostra que, se não há existência de males, todos os mercados efetivamente estarão em equilíbrio. Porém, quando bens indesejáveis estão presentes, pode haver excesso de oferta em algum mercado. Diante dessa situação, ocorre que se define mais precisamente o equilíbrio quando não há, pelo menos, excesso positivo de demanda e o vetor de preços satisfaz a condição:

$$
\sum_{i} x_{i}\left(p^{*}, p^{*} \omega_{i}\right) \leq \sum_{i} \omega_{i}
$$

Adicionalmente, o excesso de demanda pode ser dado por

$$
z(p)=\sum_{i=1}^{n}\left[x_{i}\left(p, p \omega_{i}\right)-\omega_{i}\right]
$$

O Global Trade Analysis Project (GTAP) foi estabelecido em 1992 com o objetivo de promover a pesquisa quantitativa em economia internacional através de um amplo instrumental de modelagem econômica. O componente básico do projeto consiste em sua base de dados, que contém informações relativas ao comércio bilateral, transporte, tarifas e informações insumo-produto específicas que permitem mapearem-se as interligações setoriais em cada região.

Os gastos de uma determinada região são orientados por uma função utilidade Cobb-Douglas agregada que aloca os gastos em três categorias: privados (PRIVEXP), públicos (GOVEXP) e poupança (SAVE). Na abordagem 
padrão, a função utilidade agregada assegura a participação de cada categoria na restrição orçamentária regional.

Figura 3.1 - Fluxo de renda em uma economia fechada para o modelo GTAP.

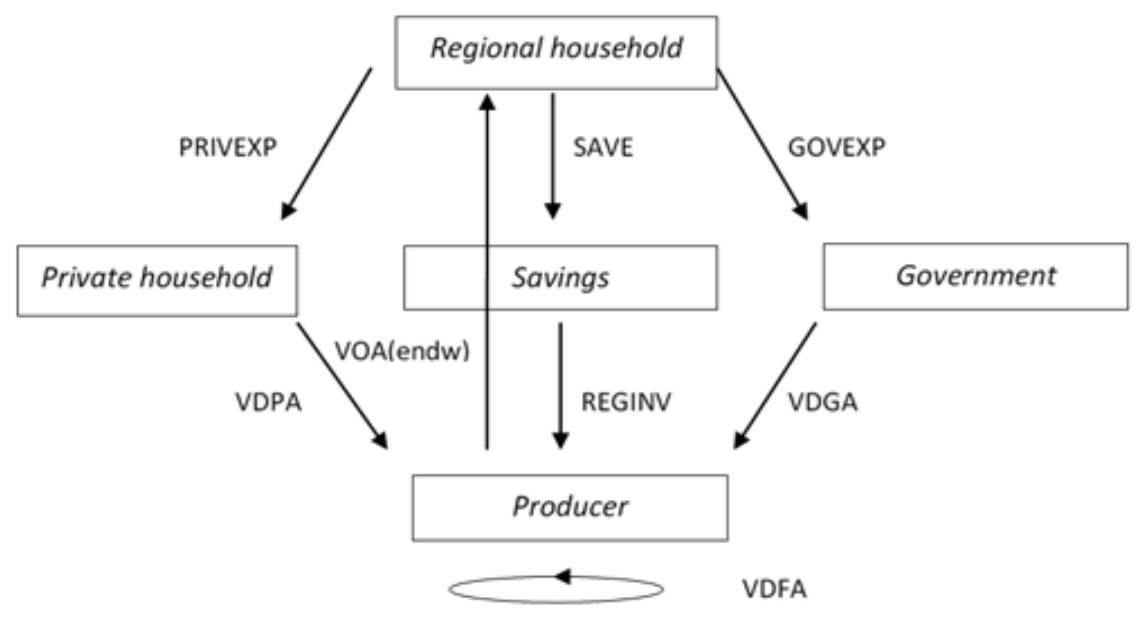

Fonte: Hertel, T. (Org.) Global Trade Analysis. 1997. p. 16.

Os fluxos da figura 3.1 assumem a ausência de taxas. A fonte de renda de determinada região (Regional household) compreende a "venda" da dotação natural de commodities (terra, capital e trabalho) para as firmas. A renda da região é representada por VOA (endw), que denota o valor do produto a preços de fatores. As firmas (Producer) combinam essa dotação de commodities com bens intermediários (VDFA - valor das compras domésticas pelas firmas a preços de fatores) para produzir bens finais. Esse processo envolve vendas às unidades familiares (Private household) e ao governo (Government), e a venda de bens de investimento (Savings) para suprir a demanda por poupança.

Ao acrescentar-se o Resto do Mundo (ROW) à análise, ainda assumindose a ausência de taxas, incorpora-se uma fonte de importações e um destino de exportações dos bens finais regionais. As importações representam pagamentos dos agentes regionais para o resto do mundo. Ao mesmo tempo, introduz-se o setor Global Bank para intermediar a poupança do resto do mundo e os investimentos regionais. O segundo setor a ser introduzido é o de comércio internacional e atividades de transporte, que contempla exportações regionais, transporte e serviços de seguros, e produz um bem composto utilizado para fazer circular o comércio entre regiões. $\mathrm{O}$ valor desse bem consiste na diferença entre o valor fob das exportações e o valor cif das importações globais. 
Figura 3.2 - Esquema de uma economia aberta no modelo GTAP.

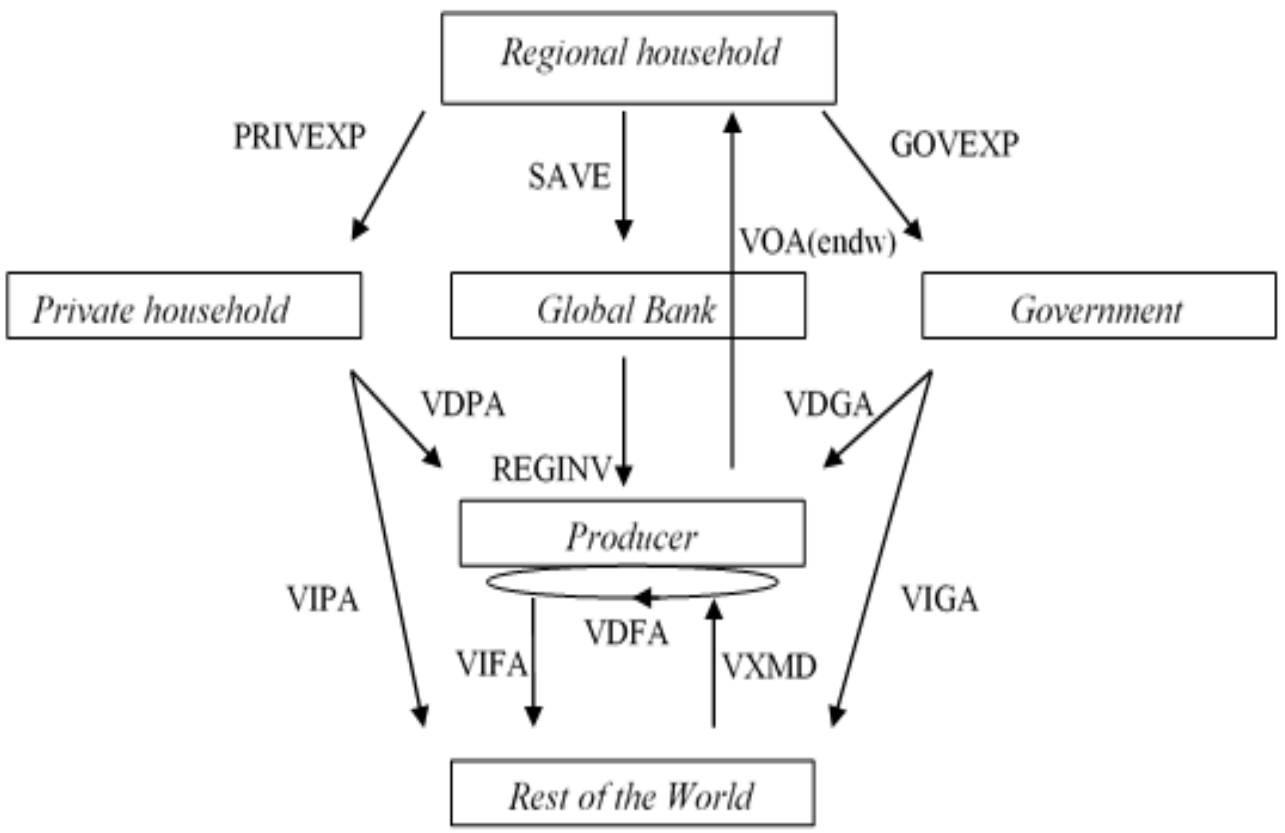

Fonte: Hertel, T. (Org.) Global Trade Analysis. 1997. p. 17.

A inclusão de taxa sobre a produção, denotada no modelo GTAP por PTAX $(i, r)$, leva ao valor da produção a preços de mercado, $\operatorname{VOM}(i, r)$. VOM deve ser igual à soma do valor das vendas domésticas a preços de mercado, $\operatorname{VDM}(i, r)$, e o valor das exportações da commodity "i” originárias da região " $r$ " a preços de mercado doméstico, destinada à região "s", $\operatorname{VXMD}(i, r, s)$.

As unidades familiares despedem sua renda em compras de bens produzidos internamente (valor das compras de unidades familiares a preços de fatores, $\operatorname{VDPA}(i, s))$ e em bens externos $(\operatorname{VIPA}(i, s))$. Deduzindo-se as taxas de importação e domésticas, $\operatorname{IPTAX}(i, s)$ e $\operatorname{DPTAX}(i, s)$, obtém-se os valores respectivos a preços de mercado, $\operatorname{VIPM}(i, s)$ e $\operatorname{VDPM}(i, s)$.

As firmas demandam insumos internos e externos, nos montantes $\operatorname{VDFA}(i, j, s)$ e $\operatorname{VIFA}(i, j, s)$. Ajustando-se as taxas intermediárias da produção, obtém-se os valores a preços de mercado, $\operatorname{VDFM}(i, j, s)$ e $\operatorname{VIFM}(i, j, s)$. As firmas também compram serviços de commodities non-tradable, referentes à dotação natural de commodities da região, fornecidas pelas unidades familiares.

Algebricamente, os gastos totais da região $r$ são dados por:

$$
\mathrm{S}[\operatorname{VPA}(i, r)+\operatorname{VGA}(i, r)+\operatorname{SAVE}(r)]
$$

Na equação 4, VPA $(i, r)$ representa o valor dos gastos privados agregados da região $r$ na commodity $i, \operatorname{VGA}(i, r)$ denota os gastos públicos totais da região $r$ na commodity $i$, e $\operatorname{SAVE}(r)$, a poupança líquida da região $r$. 
Os setores globais compreendem o setor de transportes internacionais, fornecidos pelas economias regionais e "exportados" para o setor global de transportes, e o setor de intermediação financeira internacional. Supõe-se que esse último setor fornece um bem de investimento composto, baseado em um portfólio de "investimento regional líquido", e o oferece aos demandantes internacionais de poupança.

No que diz respeito aos aspectos comportamentais das firmas, assumemse as hipóteses de retornos constantes de escala e elasticidade-substuição constante. Essas suposições resultam em uma função demanda condicional da firma em que os seus argumentos sejam apenas os preços relativos dos fatores (terra, trabalho e capital). A decisão sobre fontes de insumos - doméstica ou externa - por parte das firmas é dada pela especificação da abordagem de Armington, em um ambiente de competição perfeita, em que as firmas decidem otimamente o mix de insumos domésticos e importados. Alguns autores reconhecem que essa suposição é muito forte e que alguns setores requerem uma modelagem de competição imperfeita e de diferenciação de produtos, mas ressaltam que isso exigiria um enorme esforço em termos de informações globais referentes à concentração industrial e economias de escala (Hertel e Tsigas, 19 97).

O comportamento das households é dado por uma função utilidade agregada especifica da pela composição do consumo privado, consumo do governo e poupança. No modelo GTAP, utiliza-se uma forma específica da função-utilidade Stone-Geary, onde a participação da poupança no orçamento de subsistência é nula. Essa função permite que problemas de maximização intertemporal sejam solucionados equivalentemente através de métodos de maximização atemporal. Adicionalmente, utiliza-se um índice dos gastos correntes do governo como proxy para a estimação do bem-estar gerado pela oferta de bens e serviços públicos para as unidades privadas da região. A demanda do governo se estabelece da mesma forma que as firmas, alocando seus recursos entre bens internos e externos.

A utilidade do consumo privado é dada por uma função CDE (constant difference of elasticities). A função CDE pode ser considerada um meio-termo entre a função CES e outras funções mais flexíveis (Hertel e Tsigas, 1997). A incorporação da função CDE ao modelo GTAP deve-se à conveniência de sua calibragem diante das disponibilidades de dados sobre renda e elasticidades da demanda.

O fechamento macroeconômico do modelo, dada a natureza estática do GTAP, não contempla a hipótese de o investimento afetar a capacidade produtiva de indústrias ou regiões em períodos subseqüentes. No entanto, os deslocamentos e fluxos de investimento poderão afetar a produção e o comércio através de seus efeitos sobre a demanda final. Nesses casos, as formas de contabilização do investimento em modelos estáticos são, geralmente de cunho não-neoclássicas, onde o investimento é fixo e outra variável se ajusta automaticamente. A solução encontrada pelo GTAP consiste na exogeinização da balança comercial e endogeinização tanto da poupança quanto do investimento nacionais, ou, mais usualmente, a permissão do ajuste da poupança e do investimento globais 
através de um terceiro agente, o "global sector".

Outro importante aspecto a ser ressaltado sobre o GTAP consiste nas formas de contabilização das relações contábeis e do método de solução do modelo. O método de solução adotado pelo GTAP requer a linearização de todas as equações comportamentais e a sucessiva atualização de coeficientes para cada simulação. O método de Johansen tem sido substancialmente criticado para a solução de modelos de equilíbrio geral aplicado em virtude dos erros que gera (Hertel e Tsigas, 1997). A precisão dos modelos linearizados pode ser aprimorada através do método de solução linear de Euler. O método-padrão utilizado pelo GTAP é o método de Gragg com extrapolação, similar ao método de Euler pelas "quebras" sucessivas dos choques exógenos.

O Sistema de Projeções da Poluição Industrial (IPPS) foi desenvolvido com o objetivo de se fornecerem estimativas confiáveis e detalhadas das fontes de poluição industrial. O sistema prototípico foi construído com informações de 1987 relativas a aproximadamente 200.000 plantas industriais de todas as regiões dos EUA, abrangendo 1.500 categorias de produtos, todas as tecnologias operantes e centenas de poluentes para três categorias: ar, água e descartes sólidos, incluindo a incorporação dos fatores de riscos ecotóxicos.

As fontes primárias dos dados do IPPS consistem na Emissions Databases, da Human Health and Toxicity Database (HHDE) e da Longituginal Research Database (LRD), todas mantidas pela US Environmental Protection Agency (EPA). A Emissions Databases incorpora dados referentes a lançamentos tóxicos através do Toxic Release Inventory (TRI); qualidade do ar e emissões através do Aerometric Information Retrieval System (AIRS) e de usos da água através do National Pollutant Discharge Elimination System (NPDES). De maneira complementar, o HHDE fornece os índices de potencialidade toxicológica associados aos descartes químicos, e a LRD, as informações detalhadas das operações das plantas industriais com base em códigos SIC (Standard Industrial Classification System).

Os dados permitiram estimar um índice de intensidade de poluição por setor, dado qualquer nível de atividade de produção, expresso pela razão entre poluição por unidade de produto do setor industrial. Adicionalmente, o nível de desagregação e as informações constantes dessas bases de dados também permitem a formulação de índices complementares, inclusive com atribuição de pesos estatísticos para se contemplar o potencial toxicológico dos resíduos poluentes presentes nas emissões industriais. Da mesma forma, o IPPS ainda pode especificar a intensidade de poluição (ar, terra e água), segundo seis diferentes critérios para a poluição atmosférica e dois critérios para a poluição aquática.

Em termos de comparações internacionais, os dados referentes aos EUA sofreram novas agregações para se enquadrarem no International Standard Industrial Classification System (ISIC), reduzindo-se a 80 sub-setores.

No que diz respeito aos aspectos metodológicos, Hettige et al. (1994, p. 66) consideram que as diferenças das condições econômicas, tecnológicas e regulatórias entre países podem afetar a aplicabilidade internacional do IPPS. 
Porém, embora existam essas diferenças, o ranking relativo da intensidade de poluição entre os setores tende a permanecer constante.

\section{Gráfico 3.1 Índice Linear de Toxicidade Humana Aguda (ILTHA) por sub- setor da indústria, por código ISIC ${ }^{6}$.}

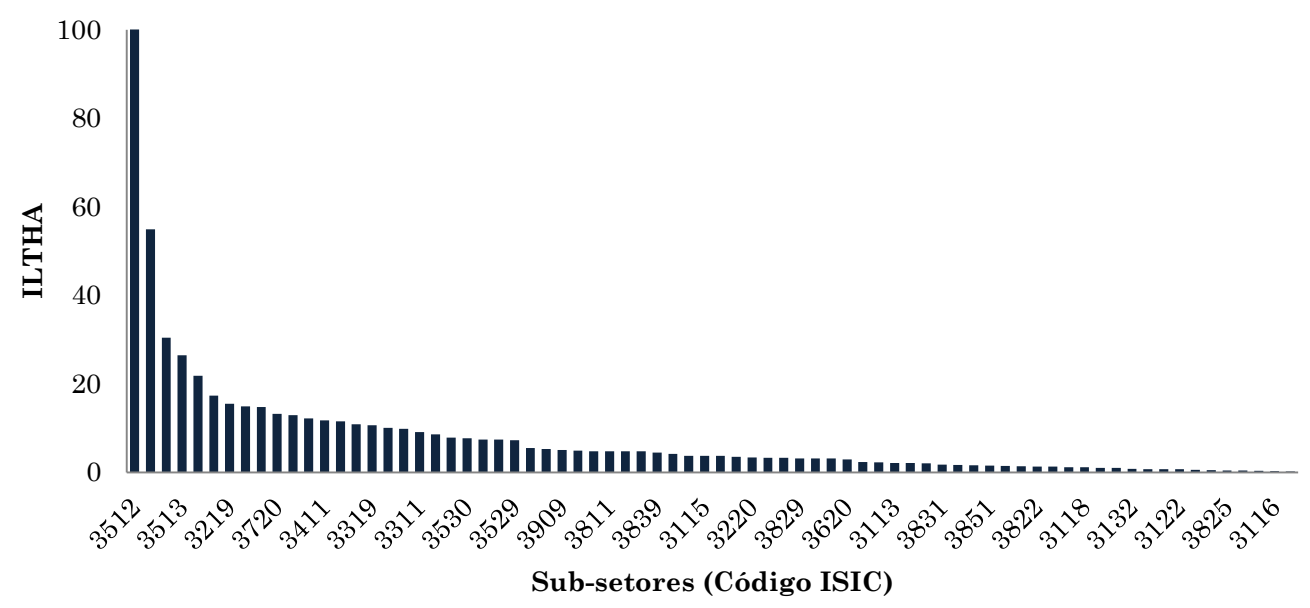

Fonte: Hettige et. al. The Industrial pollution projection system. 1994.

Neste trabalho, para fins de compatibilização com a base de dados do GTAP, procedeu-se a uma nova agregação dos setores que compõem o índice. Em virtude de a base de dados do GTAP ser menos desagregada que a classificação ISIC, foram estimados novos índices para os setores semelhantes através de média simples. Outros foram excluídos por não corresponderem perfeitamente àqueles constantes da base de dados do GTAP. Por meio disso, foram obtidos e selecionados 32 setores e seus ILTHA correspondentes. Os setores que serão utilizados neste trabalho estão dispostos na tabela 7.4 do anexo.

\section{Cenários, simulações e resultados}

As simulações são implementadas na base de dados do GTAP para avaliação dos impactos ambientais de possíveis acordos do Brasil com a União Européia e NAFTA, ou da intensificação do processo de redução de tarifas comerciais, no caso do MERCOSUL, do qual o Brasil já é uma das partes signatárias. Da mesma forma, são apresentados os resultados das simulações obtidas a partir do modelo GTAP.

O procedimento metodológico para a avaliação dos impactos ambientais dos acordos de liberalização comercial internacional consistirá na observação das atividades produtivas e a verificação de quais setores são mais intensivos em termos de degradação do meio ambiente. O modo para definição desses setores consistiu na ponderação das variações do produto dos setores da indústria pelos respectivos índices lineares de toxicidade humana aguda (ILTHA) antes e após 
a simulação de redução total de tarifas de importação e exportação entre o Brasil e os parceiros analisados. Assume-se então, neste caso, que a liberalização comercial elimina completamente as tarifas comercias.

\subsection{O Mercado Comum do Sul-MERCOSUL}

O Mercado Comum do Sul (MERCOSUL) foi firmado em 1991 por Argentina, Brasil, Paraguai e Uruguai através do Tratado de Assunção, e pode ser considerado um sucessor das discussões iniciadas na década de 1980, entre Brasil e Argentina, para o estabelecimento de um acordo de integração, cooperação e desenvolvimento entre esses dois países.

Os objetivos iniciais do Tratado de Assunção endereçavam claramente a dinamização econômica regional por meio da flexibilização do trânsito de pessoas, mercadorias e capitais intrabloco. O Tratado estabeleceu, inicialmente, uma zona de livre comércio. Em seguida, avançou para uma união aduaneira, onde os países passaram a adotar uma tarifa externa comum.

Em 1996, Bolívia e Chile adquiriram o status de Estados associados, seguidos por Peru (2003), Colômbia (2004), Equador (2004) e Venezuela (2004) através de acordos de complementação econômica entre esses países e o Mercosul. Em 2006, a Venezuela ratificou o protocolo de entrada no Mercosul e adotou os marcos regulatórios legais, políticos e comerciais do bloco. Considera-se Resto do Mercosul, neste trabalho, para fins de simulação, Argentina, Uruguai, Paraguai e Venezuela.

No que diz respeito às características econômicas, o Produto Interno Bruto (PIB), se considerados apenas os Estados-partes, incluindo a Venezuela, atinge atualmente mais de US $\$ 3$ trilhões. No entanto, a participação do PIB brasileiro corresponde a aproximadamente 70\% do PIB do Mercosul. Essa desproporção tem sido fonte de constantes divergências políticas e embates econômicos. Outra agravante é a situação comercial superavitária do Brasil com todos os demais países do bloco ao longo dos últimos anos, destacando-se sobretudo a concentração das exportações brasileiras em produtos manufaturados e importação de bens primários, favorecendo os termos de troca para a economia brasileira.

\subsubsection{Resultados}

Os resultados simulados para uma liberalização comercial total entre o Brasil e o Resto do Mercosul mostram que há uma redução efetiva da média da intensidade de poluição no caso deste acordo bilateral, e a liberalização se faz, portanto, desejável do ponto de vista ambiental. Para o Brasil, há uma redução de $0,14 \%$ da média ponderada, e para o resto dos países do Mercosul uma redução da ordem de $0,47 \%$. A tabela 4.1 resume os resultados encontrados.

Para o Brasil, entre os setores que apresentam maiores variações percentuais positivas do produto, dada a liberalização, estão os setores veículos 
e partes $(1,20 \%)$, máquinas e equipamentos (0,50\%) e têxtil (0,59\%). No caso do Resto do MERCOSUL, as maiores variações positivas se dão nos setores veículos e partes (6,21\%), máquinas e equipamentos (4,96\%) e manufaturados (0,09\%). Com exceção de poucos setores, além dos supracitados, o Resto do MERCOSIL apresenta um desempenho preponderantemente negativo, fato que pode sugerir vantagens comparativas limitadas com relação à economia brasileira.

Em termos de variação do PIB das regiões, obtém-se um crescimento de 0,05\% para o Brasil e 0,03\% para o Resto do MERCOSUL, resultantes da total liberação comercial. Esses resultados baixos refletem a incidência de baixas tarifas que já vigora dentro do bloco.

Tabela 4.1 Média ponderada do produto dos setores da indústria pelo respectivo ILTHA, pré- e pós-simulação de FTA entre Brasil e Resto do MERCOSUL.

\begin{tabular}{lccc}
\hline & Pré-simulação & Pós-simulação & Variação (\%) \\
\hline Brasil & 6,8705 & 6,8609 & $-0,1394$ \\
Resto do MERCOSUL & 6,5096 & 6,4788 & $-0,4730$ \\
\hline
\end{tabular}

Fonte: Elaboração dos autores.

\subsection{North American Free Trade Agreement - NAFTA}

O Tratado Norte-americano de Livre Comércio (NAFTA) foi formalizado em 1994 por EUA, Canadá e México, resultante de um acordo de liberalização econômica já existente entre EUA e Canadá desde 1988, que recebeu a adesão do México em 1992. O Tratado previu, inicialmente, o prazo de 15 anos para a completa eliminação de barreiras comerciais entre seus membros.

\subsubsection{Resultados}

Contrariamente à extinção de tarifas comerciais entre o Brasil e o Resto do MERCOSUL, um acordo com os países do NAFTA se mostra ambientalmente mais nocivo para o Brasil. Os resultados resumidos na tabela 4.2 mostram que há um aumento de 0,23\% na média ponderada de intensidade de poluição para o Brasil, enquanto que a média correspondente aos países do NAFTA permanece estável, com uma ligeira tendência de queda (0,002\%).

Os setores que apresentam maiores variações percentuais positivas no produto são, no Brasil, couros manufaturados (14,04\%), veículos e componentes $(3,72 \%)$ e têxtil (2,00\%); com índices de poluição de, respectivamente, 30.4, 1.19 e 15,5. No caso dos países integrantes do NAFTA as maiores variações ocorrem nos setores de equipamentos eletrônicos (0,17\%), máquinas e equipamentos $(0,14 \%)$ e químicos e plásticos $(0,11 \%)$, com índices de poluição de $3.14,3.16$ e 12.25 , respectivamente.

Ao mesmo tempo, o PIB das regiões apresenta uma variação de $0,15 \%$ e 0,003\%, para o Brasil e NAFTA, respectivamente. Esses resultados corroboram 
a maior sensibilidade da economia brasileira a um acordo com o NAFTA, e os poucos efeitos de um acordo nos países integrantes desse último bloco, possivelmente devido ao peso da economia norte-americana.

Tabela 4.2 Média ponderada do produto dos setores da indústria pelo respectivo ILTHA, pré- e pós-simulação de FTA entre Brasil e NAFTA.

\begin{tabular}{lccc}
\hline & Pré-simulação & Pós-simulação & Variação (\%) \\
\hline Brasil & 6,8705 & 6,8864 & 0,2325 \\
NAFTA & 6,4576 & 6,4577 & 0,0018 \\
\hline
\end{tabular}

Fonte: Elaboração dos autores.

\subsection{União Europeia - UE}

O ponto de partida da formação da União Européia se deu ainda em 1951 através da assinatura do Tratado de Paris, que estabeleceu a Comunidade Européia do Carvão e do Aço, composta por Alemanha, Bélgica, França, Itália, Luxemburgo e Países Baixos. Seis anos depois, o Tratado de Roma criou a Comunidade Econômica Européia (CEE). Desde então, esse bloco experimentou diversas fases de expansão, culminando com a integração de Bulgária e Romênia, em 2007, e de um total de 27 países ao longo de sua história.

Em 1962, foi estabelecida a política agrícola comum que levou aos Estados-partes o controle comum da produção alimentar e a uniformização dos preços agrícolas na Comunidade. Ainda na década de 1960 foram eliminados os direitos aduaneiros entre os seis países, e, dessa forma, constituída uma zona de livre comércio na Europa.

$\mathrm{Na}$ década seguinte, surgem as primeiras possibilidades de criação de uma moeda única e a integração de mais três países à CEE (Dinamarca, Irlanda e Reino Unido). Em 1970, os Estados-membros limitaram as margens de flutuação de suas moedas com fins de garantia à estabilidade monetária, medida fundamental para o processo de uniformização monetária, que culminou com o lançamento da moeda única (Euro) trinta anos depois. A partir dos 1980, as diferenças entre legislações nacionais começaram a criar novos obstáculos à circulação de mercadorias e daí a necessidade de harmonização legislativa. Nessa mesma década, Grécia, Portugal e Espanha aderiram à CEE.

Em 1993, assinou-se o Tratado da União Européia (conhecido como Tratado de Maastricht) onde foram estabelecidas regras e metas para a criação de uma zona monetária única e cooperação para demais formas integração institucional do bloco, agora denominado União Européia. Da mesma forma, foram estabelecidos o mercado único e a chancela das liberdades de circulação de pessoas, mercadorias, serviços (parcialmente) e capitais. Em 1995, Áustria, Finlândia e Suécia aderiram ao bloco, e, em seguida, passou-se a negociar a adesão de mais 10 países da Europa Oriental. Atualmente, o bloco é composto por 27 países.

Diante do exposto, a União Européia é considerada a forma mais 
evoluída de integração regional, não somente econômica mas também política, embora uma série de fatores ainda gerem divergências internas.

\subsubsection{Resultados}

As simulações correspondentes a um acordo de livre comércio entre o Brasil e União Europeia mostram que há um efeito ambiental líquido nocivo apenas para a EU (0,086\%). O aumento da média ponderada de intensidade de poluição mostrou-se maior que para qualquer outro acordo simulado, entretanto, ainda pequeno. $\mathrm{O}$ Brasil sofreria a maior redução no índice de poluição entre os três cenários (-1,38\%). A tabela 4.3 resume os resultados encontrados.

Em termos de variação da produção industrial setorial, as maiores variações positivas que tendem a ocorrer no Brasil são nos setores de carnes (24,81\%), derivados da carne (11,95\%), produtos da indústria alimentícia (4,04\%) e cereais em grão, que possuem índices de poluição relativamente baixos (0.7, $0.4,2.0$ e 0.2 , respectivamente). Na UE haveria aumentos mais significativos de produção de máquinas e equipamentos (0,35\%), veículos e componentes $(0,20 \%)$, indústria química $(0,15 \%)$ e metais ferrosos $(0,14 \%)$. Os índices estimados para esses setores mostram um potencial maior de poluição. São, respectivamente, $3.16,1.19,12.25$ e 12.93 .

Em termos de variação no PIB, tem-se para o Brasil e UE, 0,18\% e $0,04 \%$, respectivamente. Nota-se que a economia europeia é menos sensível a um acordo de livre comércio que o Brasil. Paralelamente, é possível perceber também que há um deslocamento da produção intensiva em capital para a UE, cujos potenciais de nocividade ambiental são mais elevados. Enquanto que no Brasil os maiores ganhos ocorrem em atividades relacionadas às atividades primárias e pouco intensivas em capital. Esse efeito pode explicar os sentidos contrários das variações percentuais dos índices.

Tabela 4.3 Média ponderada do produto dos setores da indústria pelo respectivo ILTHA, pré- e pós-simulação de FTA entre Brasil e UE.

\begin{tabular}{lccc}
\hline & Pré-simulação & Pós-simulação & Variação (\%) \\
\hline Brasil & 6,8705 & 6,7754 & $-1,3836$ \\
UE & 6,7804 & 6,7863 & 0,08630 \\
\hline
\end{tabular}

Fonte: Elaboração dos autores.

\section{Conclusões}

Os fenômenos da integração regional e o progressivo aumento da produção para sustentar a demanda da população mundial talvez sejam constantes com as quais os formuladores de políticas tenham de lidar inevitavelmente nos próximos anos. De um lado, o protecionismo procura manter a relativa estabilidade interna dos níveis de emprego, postergando os efeitos perniciosos 
da competitividade internacional à custa de um sacrifício da sociedade. Por outro, os consumidores sentem-se propensos às vantagens decorrentes do comércio internacional. Nesse contexto, insere-se ainda a discussão sobre utilização eficiente dos recursos naturais e dos demais impactos das alterações, em escala e composição, da produção industrial sobre o meio-ambiente e, em última instância, sobre a vida humana.

Neste trabalho, procurou-se avaliar a tendência estática das mudanças ocasionadas pela liberalização comercial entre Brasil, Resto do MERCOSUL, NAFTA e UE. Em relação à UE, obtiveram-se maiores variações para o Brasil em setores com menor potencial toxicológico, e o inverso para a UE. É possível identificar também alguns setores em que possivelmente o Brasil detém vantagens comparativas, principalmente setores primários da indústria, entre eles a produção de carnes, açúcar e grãos.

É possível que, no caso do NAFTA, verifique-se a HPP tal qual estabelecida na literatura, uma vez que os setores que apresentaram maiores variações para o Brasil podem ser considerados relativamente mais intensivos em poluição (couros manufaturados) que aqueles com maiores variações para o NAFTA, relativamente mais intensivos em capital (máquinas e equipamentos, químicos e plásticos e manufaturados). Esse fato levou a um aumento do índice de toxicidade humana aguda ponderado pelas variações do produto setorial da indústria no Brasil mais que proporcionalmente ao aumento do índice para economia do NAFTA, seja pelas maiores variações do produto para os setores industriais brasileiros, seja pelo maior índice de toxicidade atribuído aos setores que apresentaram as maiores variações. No caso do Mercosul, percebem-se claramente as vantagens comparativas do Brasil em setores intensivos em capital através das suas variações positivas de produto.

Nesse ponto, seria impossível generalizar uma conclusão sobre os impactos do comércio internacional sobre o meio-ambiente. No entanto, percebe-se que os seus efeitos podem variar segundo diferentes configurações de integração regional, isto é, os seus efeitos dependem sumariamente, em curto prazo, do grau com que o livre-comércio pode alterar a composição do produto nacional e a participação dos diferentes setores da indústria na produção, e, evidentemente também, do grau de intensidade de poluição demandada por cada setor. Essas alterações, no entanto, podem ser explicadas pelas teorias do comércio internacional, sobretudo pelo modelo de dotação de fatores de Hecksher-Ohlin. Nesse sentido, sugere-se que agenda de pesquisa futura inclua a expansão do número de setores analisados e maiores desagregações de dados. Da mesma forma, as análises dinâmicas poderão fornecer insights mais amplos sobre a evolução dos impactos do comércio internacional sobre o meio-ambiente no longo prazo, incorporando efeitos como a elasticidade-renda por bens ambientais e a eficiência técnica, ambos gerados pela exposição de consumidores e firmas, respectivamente, ao comércio internacional. 


\section{Referências}

Antweiler, W.; Copeland, B.; Taylor, M. (1998). "Is trade good for the environment?" NBER Working Paper Series. n. 6707. Cambridge: National Bureau of Economic Research.

Baumol, W.; Blackman, S.; Wolff, E. (1989). Productivity and american leadership: the long view. Cambridge: MIT Press.

Bommer, R. (1999). "Environmental policy and industrial competitiveness: the pollution-haven hypothesis reconsidered." Review of International Economics, 7(2), p. 342-355.

Eskeland, G.; Harrison, A. (1997). "Moving to greener pasture? Multinationals and the pollution-haven hypothesis." Policy Research Working Papers, n. 1744. Washington: World Bank.

Grossman, G.; Krueger, A. (1991). "Environmental impacts of a north american free trade agreement." NBER Working Paper. n. 3914. November.

Grossman, G.; Krueger, A. (1993). "Environmental impacts of a north american free trade asgreement." In: Garber, P. The Mexico-US free trade agreement. Cambridge: MIT Press.

Hansen, W.; Prusa, T. (1997). "The economics and politics of trade policy: an empirical analysis of ITC decision making." Review of International Economics. 5(2), p. 230-245.

Hecksher, E. (1950). "The effect of foreign trade on the distribution of income." In: Readings on International Trade Theory. Philadelphia: Blakiston.

Helpman, E.; Krugman, P. (1995). Market structure and foreign trade. Cambridge, MIT Press.

Hertel, T.; Tsigas, M. (1997). "Structure of GTAP.” In: HERTEL, T. W. (Org.) Global trade analysis: modeling and applications. New York: Cambridge University Press.

Intergovernmental Panel on Climate Change (IPCC) (2001). Climate change 2001: the scientific basis. Contribution of working group I to the third assessment report of the IPCC. New York: Cambridge University Press.

Krugman, P. (1991). “Is bilateralism bad?” In: Helpman, E.; Razin, A. (ed.) International trade and trade policy. Cambridge: MIT Press.

Krugman, P.; Obstfeld, R. (2003). International economics. New York: Pearson.

Long, B. (2000). International environmental issues and the OCDE 1950-2000: a historical perspective. Paris: OCDE.

Mckenzie, L. (1989). “General equilibrium.” In: The New Palgrave: general equilibrium. UK: Macmillan Press.

Ohlin, B. (1933) Interregional and international trade. Cambridge: Harvard University Press.

Oladi, R.; Beladi, H. (2008). "Is regionalism viable? A case for global free trade." Journal of International Economics. 16(2), p. 293-300. 
Samuelson, P. (1948). "International trade and the equalisation of factor prices." Economic Journal. 58, p. 163-184.

Samuelson, P. (1949). "International factor-price equalisation once again." Economic Journal. 59, p. 181-197.

United Nations Environmente Programme (UNEP) (2007). Human vulnerability to environmental change. UNEP.

United Nations Environment Programme (UNEP); United Nations Comission on Trade and Development (UNCTAD). (1974). Patterns of resource use, environment and development strategies. Conferência UNEP/UNCTAD. Cocoyc, México. Junho.

Varian, H. (1992). Microeconomic analysis. 3. ed. New York: W.W. Norton.

Walras, L. (1996). "Compêndio dos elementos de economia política pura.” Trad. João Guilherme Vargas Neto. In: Os Economistas. São Paulo: Nova Cultural.

World Bank (1995). "The industrial pollution projection system.” Policy Research Working Papers, n. 1431. Washington: World Bank.

World Bank (2000). Trade blocs. Oxford: Oxford University Press,.

World Trade Organization (WTO). World trade report 2007 Six decades of multilateral trade cooperation: what have we learnt? Switzerland: WTO Publications, 2007.

World Trade Organization (WTO). Trade and environment. (1999). Special studies. Switzerland: WTO Publications.

Xing, Y.; Kolstad, K. (1998). Do lax environmental regulations attract foreign investment? Santa Barbara: University of California.

\section{Anexos}

7.1 Lista de setores da indústria e códigos ISIC.

\begin{tabular}{|l|c|}
\hline FERTILIZERS \& PESTICIDES & 3512 \\
\hline INDUSTRIAL CHEMICALS EXCEPT FERTILIZER & 3511 \\
\hline TANNERIES AND LEATHER FINISHING & 3231 \\
\hline SYNTHETIC RESINS, PLASTICS MATERIALS, \& MANMADE FIBRES & 3513 \\
\hline PAPER \& PAPERBOARD CONTAINERS \& BOXES & 3412 \\
\hline PLASTICS PRODUCTS, N.E.C. & 3560 \\
\hline TEXTILES, N.E.C. & 3219 \\
\hline PRINTING \& PUBLISHING & 3420 \\
\hline PULP, PAPER \& PAPERBOARD ARTICLES & 3419 \\
\hline NONFERROUS METALS & 3720 \\
\hline IRON AND STEEL & 3710 \\
\hline RUBBER PRODUCTS, N.E.C. & 3559 \\
\hline PULP, PAPER, \& PAPERI3OARD & 3411 \\
\hline FABRICATED METAL PRODUCTS & 3819 \\
\hline MUSICAL INSTRUMENTS & 3902 \\
\hline
\end{tabular}




\begin{tabular}{|c|c|}
\hline WOOD \& CORK PRODUCTS, N.E.C. & 3319 \\
\hline FURNITURE \& FIXTURES, NONMETAL & 3320 \\
\hline PAINTS, VARNISHES, \& LACQUERS & 3521 \\
\hline SAWMILLS, PLANING \& OTHER WOOD MILLS & 3311 \\
\hline STRUCTURAL METAL PRODUCTS & 3813 \\
\hline NONMETALLIC MINERAL PRODUCTS. N.E.C. & 3699 \\
\hline PETROLEUM REFINERIES & 3530 \\
\hline DRUGS AND MEDICINES & 3522 \\
\hline SPINNING, WEAVING, \& FINISHING TEXTILES & 3211 \\
\hline CHEMICAL PRODUCTS, N.E.C. & 3529 \\
\hline POTTERY, CHINA, \& EARTHENWARE & 3610 \\
\hline METAL \& WOOD WORKING MACHINERY & 3823 \\
\hline MANUFACTURING INDUSTRIES, N.E.C. & 3909 \\
\hline MADE-UP TEXTILES EXCEPT APPAREL & 3212 \\
\hline MISC. PETROLEUM \& COAL PRODUCTS & 3540 \\
\hline CUJTLERY, HAND TOOLS, \& GENERAL HARDWARE & 3811 \\
\hline KNITTING MILLS & 3213 \\
\hline WATCHES AND CLOCKS & 3853 \\
\hline ELECTRICAL APPARATUS AND SUPPLIES, N.E.C. & 3839 \\
\hline JEWELRY AND RELATED ARTICLES & 3901 \\
\hline SHIPBUILDING AND REPAIRING & 3841 \\
\hline OILS AND FATS & 3115 \\
\hline FURNITURE \& FIXTURES OF METAL & 3812 \\
\hline SOAP, CLEANING PREPS., PERFUMES, \& TOILET PREPS. & 3523 \\
\hline WEARING APPAREL & 3220 \\
\hline FOOTWEAR & 3240 \\
\hline SPORTING AND ATHLETIC GOODS & 3903 \\
\hline MACHINERY \& EQUIPMENT, N.E.C. & 3829 \\
\hline RADIO, TV, \& COMMUNICATION EQUIPMENT & 3832 \\
\hline ENGINES AND TURBINES & 3821 \\
\hline GLASS AND GLASS PRODUCTS & 3620 \\
\hline ELECTRICAL APPLIANCES \& HOUSEWARES & 3833 \\
\hline DAIRY PRODUCTS & 3112 \\
\hline PRESERVED FRUITS \& VEGETABLES & 3113 \\
\hline AIRCRAFT & 3845 \\
\hline FOOD PRODUCTS, N.E.C. & 3121 \\
\hline ELECTRICAL INDUSTRIAL MACHINERY & 3831 \\
\hline RAILROAD EQUIPMENT & 3842 \\
\hline PHOTOGRAPHIC AND OPTICAL GOODS & 3852 \\
\hline PROFESSIONAL \& SCIENTIFIC EQUIPMENT & 3851 \\
\hline SPECIAL INDUSTRIAL MACHINERY \& EQUIPMENT & 3824 \\
\hline
\end{tabular}




\begin{tabular}{|l|c|}
\hline STRUCTURAL CLAY PRODUCTS & 3691 \\
\hline AGRICULTURAL MACHINERY \& EQUIPMENT & 3822 \\
\hline CARPETS AND RUGS & 3214 \\
\hline MOTOR VEHICLES & 3843 \\
\hline SUGAR FACTORIES \& REFINERIES & 3118 \\
\hline CEMENT, LIME, AND PLASTER & 3692 \\
\hline TOBACCO MANUFACTURES & 3140 \\
\hline WINE INDUSTRIES & 3132 \\
\hline TIRES AND TUBES & 3551 \\
\hline BAKERY PRODUCTS & 3117 \\
\hline PREPARED ANIMAL FOODS & 3122 \\
\hline DISTILLED SPIRITS & 3131 \\
\hline CONFECTIONERY PRODUCTS & 3119 \\
\hline OFFICE, COMPUTING, \& ACCOUNTING MACHINERY & 3825 \\
\hline MEAT PRODUCTS & 3111 \\
\hline MALT LIQUORS AND MALT & 3133 \\
\hline GRAIN MILL PRODUCTS & 3116 \\
\hline SOFT DRINXS \& CARBONATED WATER & 3134 \\
\hline
\end{tabular}


7.2 Produto da indústria por setor pré- e pós-simulação para Brasil e Resto do Mercosul, em US\$ milhões. ${ }^{1}$

\begin{tabular}{|c|c|c|c|c|}
\hline \multicolumn{5}{|c|}{ Industry output of commodity $\mathrm{i}$ in region $\mathrm{r}$} \\
\hline & \multicolumn{2}{|c|}{ Pre-sim } & \multicolumn{2}{|c|}{ Post-sim } \\
\hline & Brazil & Rmerc & Brazil & Rmerc \\
\hline Wheat & 238,37 & 3195,94 & 237,8 & 3154,29 \\
\hline Cereal grains & 2401,12 & 4278,11 & 2389,38 & 4252,5 \\
\hline Vegetables, fruits, nuts & 1826,56 & 6488,23 & 1820,35 & 6465,22 \\
\hline Sugar cane, sugar beet & 3189,42 & 307,59 & 3176,24 & 307,21 \\
\hline Forestry & 1263,22 & 847,33 & 1260,34 & 841,37 \\
\hline Minerals, nec & 6831,73 & 2445,7 & 6807,18 & 2438,57 \\
\hline Meat: cattle, sheep, goats, horse & 9384,4 & 9990,34 & 9340,32 & 9947,08 \\
\hline Meat products, nec & 4665,59 & 2054,44 & 4606,15 & 2041,98 \\
\hline Vegetables oils and fats & 6707,53 & 4830,19 & 6679,58 & 4821,12 \\
\hline Dairy products & 6881,3 & 6981,33 & 6882,87 & 6969,16 \\
\hline Sugar & 1316,34 & 513,85 & 1316,49 & 510,36 \\
\hline Food products, nec & 4847,46 & 1466 & 4814,37 & 1461,49 \\
\hline Beverages and tobacco products & 28139,67 & 26026,91 & 28071,53 & 25914,93 \\
\hline Textiles & 6559,53 & 11473,13 & 6560,1 & 11471,52 \\
\hline Wearing apparel & 11751 & 6341,48 & 11807 & 6233,89 \\
\hline Leather products & 6478,29 & 7432,08 & 6492,29 & 7389,63 \\
\hline Wood products & 4981,71 & 5768,88 & 4903,26 & 5651,33 \\
\hline Paper products, publishing & 6351,3 & 6009,61 & 6305,44 & 5983,71 \\
\hline Petroleum, coal products & 17588,14 & 12107,77 & 17495,16 & 12042,08 \\
\hline Chemical, rubber plastic products & 17384,97 & 11918,55 & 17357,31 & 11854,94 \\
\hline Mineral products & 42657,77 & 29799,15 & 42580,52 & 29563,25 \\
\hline Ferrous metals & 11240,18 & 8303,84 & 11256,05 & 8309,12 \\
\hline Metals, nec & 7000,38 & 3441,75 & 6911,96 & 3348,62 \\
\hline Metal products & 14987,6 & 6353,23 & 15058,56 & 6343,63 \\
\hline Motor vehicles and parts & 17813,41 & 12227,79 & 18115,55 & 13443,16 \\
\hline Transport equipment & 14761,67 & 2358,04 & 14584,8 & 2335,34 \\
\hline Electronic equipment & 12207,58 & 3404,53 & 12163,12 & 3387,58 \\
\hline Machinery and equipment, nec & 27385,63 & 6513,77 & 27562,34 & 6936,33 \\
\hline Manufactures, nec & 10626,36 & 5376,95 & 10604,47 & 5392,56 \\
\hline Electricity & 19720,34 & 12282,92 & 19672,94 & 12225,36 \\
\hline Air transport & 3568,45 & 6910,6 & 3558,63 & 6851,4 \\
\hline Communication & 10052,18 & 13274,26 & 10044,5 & 13253,19 \\
\hline
\end{tabular}

Fonte: GTAP.

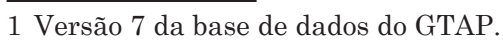


7.3 Produto da indústria por setor pré- e pós-simulação para Brasil e NAFTA, em US\$ milhões.

\begin{tabular}{|c|c|c|c|c|}
\hline \multicolumn{5}{|c|}{ Industry output of commodity $i$ in region $r$} \\
\hline & \multicolumn{2}{|c|}{ Pre-sim } & \multicolumn{2}{|c|}{ Post-sim } \\
\hline & Brazil & NAFTA & Brazil & NAFTA \\
\hline Wheat & 238,37 & 10128,84 & 228,46 & 10122,12 \\
\hline Cereal grains & 2401,12 & 26512,58 & 2365 & 26510,92 \\
\hline Vegetables, fruits, nuts & 1826,56 & 37040,57 & 1805,22 & 37036,45 \\
\hline Sugar cane, sugar beet & 3189,42 & 3753,99 & 3189,22 & 3738,9 \\
\hline Forestry & 1263,22 & 33853,2 & 1257,69 & 33836,48 \\
\hline Minerals, nec & 6831,73 & 50147,85 & 6767,1 & 50146,76 \\
\hline Meat: cattle, sheep, goats, horse & 9384,4 & 104368,1 & 9359,61 & 104311,4 \\
\hline Meat products, nec & 4665,59 & 97491,71 & 4475,16 & 97496,45 \\
\hline Vegetables oils and fats & 6707,53 & 22972,57 & 6613,28 & 22972,69 \\
\hline Dairy products & 6881,3 & 96508,34 & 6947,84 & 96452,85 \\
\hline Sugar & 4847,46 & 35109,96 & 4960,12 & 34936,24 \\
\hline Food products, nec & 28139,67 & 359687,2 & 27959,96 & 359654,1 \\
\hline Beverages and tobacco products & 6559,53 & 186310,4 & 6553,42 & 186310,8 \\
\hline Textiles & 11751 & 174647,6 & 12118,02 & 174336 \\
\hline Wearing apparel & 6478,29 & 135425,5 & 6606,04 & 135218,3 \\
\hline Leather products & 4981,71 & 25323,98 & 5902,83 & 25071,92 \\
\hline Wood products & 6351,3 & 273986,7 & 6296,17 & 273801 \\
\hline Paper products, publishing & 17588,14 & 454861,9 & 17200,79 & 454951,4 \\
\hline Petroleum, coal products & 17384,97 & 176210,4 & 17358,11 & 176169,5 \\
\hline Chemical, rubber plastic products & 42657,77 & 820681,5 & 41702,44 & 821877,6 \\
\hline Mineral products & 11240,18 & 155274,1 & 11333,18 & 155287,5 \\
\hline Ferrous metals & 17028,04 & 180860,7 & 16711,72 & 180856,3 \\
\hline Metals, nec & 7000,38 & 133632,4 & 6718,11 & 133660,8 \\
\hline Metal products & 14987,6 & 326326,8 & 14746,48 & 326426 \\
\hline Motor vehicles and parts & 17813,41 & 578423,1 & 18581,35 & 577941,1 \\
\hline Transport equipment & 14761,67 & 212913,9 & 14542,59 & 212551,1 \\
\hline Electronic equipment & 12207,58 & 415049,8 & 12078,51 & 415717,3 \\
\hline Machinery and equipment, nec & 27385,63 & 887308,6 & 26021,19 & 889095 \\
\hline Manufactures, nec & 10626,36 & 77275,86 & 10482,47 & 77295,84 \\
\hline Electricity & 19720,34 & 290971,3 & 19537,43 & 291007,2 \\
\hline Air transport & 3568,45 & 203984,3 & 3542,53 & 203862,3 \\
\hline Communication & 10052,18 & 424150,7 & 10014,73 & 424087,1 \\
\hline
\end{tabular}

Fonte: GTAP. 
7.4 Produto da indústria por setor pré- e pós-simulação para Brasil e UE, em US\$ milhões.

\begin{tabular}{|c|c|c|c|c|}
\hline \multicolumn{5}{|c|}{ Industry output of commodity $i$ in region $r$} \\
\hline & \multicolumn{2}{|c|}{ Pre-sim } & \multicolumn{2}{|c|}{ Post-sim } \\
\hline & Brazil & $E U$ & Brazil & $E U$ \\
\hline Wheat & 238,37 & 17113,02 & 191,63 & 17047,03 \\
\hline Cereal grains & 2401,12 & 18686,82 & 2614,94 & 18449,98 \\
\hline Vegetables, fruits, nuts & $1.826,56$ & 56663,07 & 1681,02 & 56757,2 \\
\hline Sugar cane, sugar beet & 3189,42 & 5023,7 & 3024,4 & 4978,19 \\
\hline Forestry & 1263,22 & 23527,25 & 1294,41 & 23509,76 \\
\hline Minerals, nec & 6831,73 & 26118,29 & 6503,36 & 26199,35 \\
\hline Meat: cattle, sheep, goats, horse & 9384,4 & 68786,99 & 22092,09 & 51700,15 \\
\hline Meat products, nec & 4665,59 & 113702,2 & 4419,1 & 112699,2 \\
\hline Vegetables oils and fats & 6707,53 & 49976,02 & 6319,03 & 49905,23 \\
\hline Dairy products & 6881,3 & 121562,6 & 6810,83 & 121505,1 \\
\hline Sugar & 1316,34 & 3785,48 & 1303,24 & 3777,54 \\
\hline Food products, nec & 4847,46 & 25579,44 & 4786,4 & 25278,82 \\
\hline Beverages and tobacco products & 28139,67 & 303723,8 & 28791,25 & 302040,3 \\
\hline Textiles & 6559,53 & 174711,6 & 6543,11 & 174797,1 \\
\hline Wearing apparel & 11751 & 146002,7 & 10858,9 & 146700,3 \\
\hline Leather products & 6478,29 & 112336,8 & 6363,18 & 112657,8 \\
\hline Wood products & 4981,71 & 59718,09 & 3976,02 & 60566,39 \\
\hline Paper products, publishing & 6351,3 & 158022 & 5627,38 & 158545,1 \\
\hline Petroleum, coal products & 17588,14 & 364652,8 & 16305,27 & 365212,3 \\
\hline Chemical, rubber plastic products & 17384,97 & 149745,2 & 16934,71 & 149832,4 \\
\hline Mineral products & 42657,77 & 818081,4 & 38410,08 & 821739,2 \\
\hline Ferrous metals & 11240,18 & 245574,6 & 10845,16 & 246063,6 \\
\hline Metals, nec & 7000,38 & 123509,8 & 5760,58 & 124245,6 \\
\hline Metal products & 14987,6 & 329304,2 & 13435,4 & 330544,4 \\
\hline Motor vehicles and parts & 17813,41 & 539269,8 & 16102,48 & 541801,8 \\
\hline Transport equipment & 14761,67 & 121155,5 & 12433,92 & 121732,5 \\
\hline Electronic equipment & 12207,58 & 401108,4 & 10972,02 & 403649,6 \\
\hline Machinery and equipment, nec & 27385,63 & 764195,2 & 22287,2 & 770770,1 \\
\hline Manufactures, nec & 10626,36 & 229336,7 & 10016,24 & 229959,7 \\
\hline Electricity & 19720,34 & 270801,7 & 18988,47 & 270947 \\
\hline Air transport & 3568,45 & 116445 & 3491,92 & 116497,4 \\
\hline Communication & 10052,18 & 263219,9 & 9912,26 & 263371,5 \\
\hline
\end{tabular}

Fonte: GTAP. 


\subsection{Lista de choques realizados no GTAP (Shocklist).}

7.5.1 Simulação Brasil - Resto do Mercosul

Shock tms(TRAD_COMM,"Brasil","RMERC") = target\% 0 from file tms.shk; Shock tms(TRAD_COMM," RMERC “,"Brasil”) = target\% 0 from file tms.shk; Shock txs(TRAD_COMM,"Brasil","RMERC") = target\% 0 from file txs.shk; Shock txs(TRAD_COMM,” RMERC “,"Brasil”) = target\% 0 from file txs.shk;

\subsubsection{Simulação Brasil - NAFTA}

Shock tms(TRAD_COMM,"Brasil","NAFTA") = target\% 0 from file tms.shk; Shock tms(TRAD_COMM," NAFTA “,"Brasil”) = target\% 0 from file tms.shk; Shock txs(TRAD_COMM,"Brasil","NAFTA") = target\% 0 from file txs.shk; Shock txs(TRAD_COMM," NAFTA “,"Brasil”) = target\% 0 from file txs.shk;

\subsubsection{Simulação Brasil - UE}

Shock tms(TRAD_COMM,"Brasil","EU") = target\% 0 from file tms.shk; Shock tms(TRAD_COMM,"EU","Brasil") = target\% 0 from file tms.shk; Shock txs(TRAD_COMM,"Brasil","EU") = target\% 0 from file txs.shk; Shock txs(TRAD_COMM,"EU","Brasil") = target\% 0 from file txs.shk; 